\title{
P02.146. Mindfulness based stress reduction in adults with mild cognitive impairment: a pilot study using fMRI
}

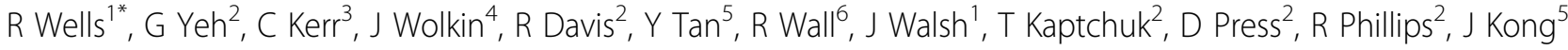 \\ From International Research Congress on Integrative Medicine and Health 2012 \\ Portland, Oregon, USA. 15-18 May 2012
}

\section{Purpose}

Fifty percent of adults with mild cognitive impairment (MCI) develop Alzheimer's disease (AD) within 5 years. Preliminary data suggest that mindfulness-based stress reduction (MBSR) increases gray matter density of the hippocampus, which atrophies in AD. We studied the safety, feasibility, and impact of MBSR on brain function, memory, and quality of life (QOL) among adults with MCI.

\section{Methods}

We randomized $14 \mathrm{MCI}$ patients (2:1) to 8 weeks of standardized MBSR $(n=9)$ or wait-list control $(n=5)$. Brain activity with fMRI resting state, neuropsychological and QOL measures were assessed at baseline and 8 weeks; the latter two were also assessed at 6 months.

\section{Results}

The mean (SD) age was 74 (7); baseline Mini-Mental State Exam score was 27.2 (1.5); class attendance was $88 \%$; and home practice was 26.1 minutes/day (19.6). No adverse events were reported. At 8 weeks, episodic memory was not improved in adults randomized to MBSR compared to the control (median change [Q1, Q3] from baseline, Rey Auditory Verbal Learning Test total recall $-2.5[-5.5,0]$ vs. $+1[-1,4], \mathrm{p}=.24)$. Non-significant trends that suggested improvement with MBSR were detected for change from baseline for MBSR vs. control for AD Assessment Scalecognitive subscale $(-0.5,[-4,0.5]$ vs. $0[-1,2], \mathrm{p}=0.46)$; Resilience Scale $(+7[2,21]$ vs. $-2[-9,0], \mathrm{p}=0.18)$; QOL-AD $(+2$ $[-1,3]$ vs. $0[-1,0], \mathrm{p}=0.25)$; Perceived Stress Scale $(-1$ $[-6,0]$ vs. $0[-4,2], \mathrm{p}=.46)$. Compared with the control group, the regional homogeneity of the left hippocampus and putamen/inferior frontal gyrus were significantly enhanced in the MBSR group. Data for 6 months are not yet available.

\section{Conclusion}

MBSR was associated with changes in spontaneous brain activity in the hippocampus and putamen/inferior frontal gyrus in adults with MCI. A randomized controlled trial in MCI evaluating effects of MBSR on neuropsychological, behavioral, and neuroimaging measures is feasible and safe.

\section{Author details}

'Brigham and Women's Hospital/Harvard Medical School, Brookline, USA. ${ }^{2}$ Beth Israel Deaconess Medical Center/Harvard Medical School, Boston, USA. ${ }^{3}$ Brown University, Providence, USA. ${ }^{4}$ NYU Langone Medical Center, New York City, USA. ${ }^{5}$ Massachusetts General Hospital/Harvard Medical School, Boston, USA. ${ }^{6}$ Commonwealth Care Alliance, Boston, USA.

Published: 12 June 2012

doi:10.1186/1472-6882-12-S1-P202

Cite this article as: Wells et al:: P02.146. Mindfulness based stress

reduction in adults with mild cognitive impairment: a pilot study using fMRI. BMC Complementary and Alternative Medicine 2012 12(Suppl 1):P202.

'Brigham and Women's Hospital/Harvard Medical School, Brookline, USA Full list of author information is available at the end of the article

(c) 2012 Wells et al; licensee BioMed Central Ltd. This is an Open Access article distributed under the terms of the Creative Commons Attribution License (http://creativecommons.org/licenses/by/2.0), which permits unrestricted use, distribution, and reproduction in any medium, provided the original work is properly cited. 